\title{
GLOBAL COMPETITIVE RANKING FOR CONSTRAINTS HANDLING WITH MODIFIED DIFFERENTIAL EVOLUTION
}

\author{
Md. Abul Kalam Azad and Edite M.G.P. Fernandes \\ Algoritmi R\&D Center, School of Engineering \\ University of Minho, 4710-057 Braga, Portugal \\ \{akazad,emgpf\}@dps.uminho.pt
}

Keywords: Constrained nonlinear programming; Constraints handling; Ranking; Differential evolution.

\begin{abstract}
Constrained nonlinear programming problems involving a nonlinear objective function with inequality and/or equality constraints introduce the possibility of multiple local optima. The task of global optimization is to find a solution where the objective function obtains its most extreme value while satisfying the constraints. Depending on the nature of the involved functions many solution methods have been proposed. Most of the existing population-based stochastic methods try to make the solution feasible by using a penalty function method. However, to find the appropriate penalty parameter is not an easy task. Population-based differential evolution is shown to be very efficient to solve global optimization problems with simple bounds. To handle the constraints effectively, in this paper, we propose a modified constrained differential evolution that uses self-adaptive control parameters, a mixed modified mutation, the inversion operation, a modified selection and the elitism in order to progress efficiently towards a global solution. In the modified selection, we propose a fitness function based on the global competitive ranking technique for handling the constraints. We test 13 benchmark problems. We also compare the results with the results found in literature. It is shown that our method is rather effective when solving constrained problems.
\end{abstract}

\section{INTRODUCTION}

Problems involving global optimization over continuous spaces are ubiquitous throughout the scientific community. Many real world problems are formulated as mathematical programming problems involving continuous variables with linear/nonlinear objective function and constraints. The constraints can be of inequality and equality type. Generally, the constrained nonlinear programming problems are formulated as follows:

$$
\begin{array}{lll}
\operatorname{minimize} & f(\mathbf{x}) & \\
\text { subject to } & g_{k}(\mathbf{x}) \leq 0 & k=1,2, \ldots, m_{1} \\
& h_{l}(\mathbf{x})=0 & l=1,2, \ldots, m_{2} \\
& l_{j} \leq x_{j} \leq u_{j} & j=1,2, \ldots, n,
\end{array}
$$

where, $f, g_{k}, h_{l}: \mathbb{R}^{n} \longrightarrow \mathbb{R}$ with feasible set $\mathcal{F}=\{\mathbf{x} \in$ $\mathbb{R}^{n}: \mathbf{g}(\mathbf{x}) \leq 0, \mathbf{h}(\mathbf{x})=0$ and $\left.\mathbf{l} \leq \mathbf{x} \leq \mathbf{u}\right\} . f, g_{k}, h_{l}$ may be differentiable and the information about derivatives may or may not be provided.

Problem (1) involving global optimization (here we consider a minimization problem) of a multivariate function with constraints is widespread in the mathematical modeling of real world systems. Many problems can be described only by nonlinear relationships, which introduce the possibility of multiple local minima. The task of the global optimization is to find a solution where the objective function obtains its most extreme value, the global minimum, while satisfying the constraints.

In the last decades, many stochastic solution methods with different constraints handling techniques have been proposed to solve (1). Stochastic methods involve a random sample of solutions and the subsequent manipulation of the sample to find good local (and hopefully global) minima. The stochastic methods can be based on a point-to-point search or on a population-based search. Most of the existing population-based stochastic methods try to make the solution feasible by repairing the infeasible one or penalizing an infeasible solution with a penalty function method. However, to find the appropriate penalty parameter is not an easy task. Deb (2000) proposed an efficient constraints handling technique for genetic algorithms based on the feasibility and dominance rules. The author used a penalty function that does not require any penalty parameter. Barbosa and Lemonge (2003) proposed a parameter-less 
adaptive penalty scheme for genetic algorithms applied to constrained optimization problems. In the very recent paper the authors proposed this adaptive penalty scheme for differential evolution (Silva, Barbosa and Lemonge, 2011). Hedar and Fukushima (2006) proposed a filter simulated annealing method for constrained continuous global optimization problems. The authors used the filter method (Fletcher and Leyffer, 2002) rather than the penalty method to handle the constraints. Runarsson and Yao proposed a stochastic ranking (Runarsson and Yao, 2000) and a global competitive ranking (Runarsson and Yao, 2003) technique for constrained evolutionary optimization based on evolution strategy. The authors presented a new view on the usual penalty function methods in terms of the dominance of penalty and objective functions. Dong, Tang, $\mathrm{Xu}$ and Wang (2005) proposed a swarm optimization based on constraint fitness priority-based ranking technique. Zahara and $\mathrm{Hu}$ (2008) proposed constrained optimization with a hybrid Nelder-Mead simplex method and a particle swarm optimization. The authors also used constraint fitness priority-based ranking technique for constraints handling. Rocha and Fernandes proposed the feasibility and dominance rules (Rocha and Fernandes, 2008) and the self-adaptive penalties (Rocha and Fernandes, 2009) in the electromagnetism-like algorithm for constrained global optimization problems. Coello Coello (2000) proposed constraints handling using an evolutionary multiobjective optimization technique. This author introduced the concept of nondominance (commonly used in multiobjective optimization) as a way to incorporate constraints into the fitness function of a genetic algorithm. Coello Coello and Cortés (2004) proposed hybridizing a genetic algorithm with an artificial immune system for global optimization. The authors used genotypic-based distances to move from infeasible solution to feasible one. Another constraints handling technique is the multilevel Pareto ranking based on the constraints matrix (Ray and Tai, 2001; Ray and Liew, 2003). This technique is based on the concepts of Pareto nondominance in multiobjective optimization. Ray and Tai (2001) proposed an evolutionary algorithm with a multilevel pairing strategy and Ray and Liew (2003) proposed a society and civilization algorithm based on the simulation of social behaviour.

Differential evolution (DE) proposed by Storn and Price (1997) is a population-based heuristic approach that is very efficient to solve global optimization problems with simple bounds. DE's performance depends on the amplification factor of differential variation and crossover control parameter. Hence selfadaptive control parameters have been implemented in DE in order to obtain a competitive algorithm. Further, to improve solution accuracy, techniques that are able to exploit locally certain regions, detected in the search space of the problem as promising, are also required. A local search starts from a candidate solution and then iteratively moves to a neighbour solution. Typically, every candidate solution has more than one neighbour solutions and the choice of movement depends only on the information about the solutions in the neighbourhood of the current one. When the solutions ought to be restricted to a set of equality and inequality constraints, an efficient constraints handling technique is also required in the solution method. In this paper, we propose a modified constrained differential evolution algorithm (herein denoted as $\mathrm{m}-\mathrm{CDE}$ ) that uses self-adaptive control parameters (Brest, Greiner, Bošković, Mernik and Žumer, 2006), a mixture of modified mutations (Kaelo and Ali, 2006), and also includes the inversion operation, a modified selection and the elitism to be able to progress efficiently towards a global solution of problems (1). To handle the constraints effectively, the modified selection incorporates the global competitive ranking technique to assess the fitness of all individual points in the population.

The organization of this paper is as follows. We describe the constraints handling techniques in Section 2. In Section 3 the modified constrained differential evolution is outlined. Section 4 describes the experimental results and finally we draw the conclusions of this study in Section 5.

\section{CONSTRAINTS HANDLING TECHNIQUES}

Stochastic methods are mostly developed for the global optimization of unconstrained problems. Finally, they are extended to the constrained problems with the modification of solution procedures or by applying penalty function methods. In populationbased techniques, the widely used approach to deal with constrained optimization problems is based on penalty functions. In penalty functions, a penalty term is added to the objective function in order to penalize the constraint violation. This enable us to transform a constrained optimization problem into a sequence of unconstrained subproblems, whose objective function is

$$
\psi(\mathbf{x})=f(\mathbf{x})+\mu H[\zeta(\mathbf{x})],
$$

where $\zeta(\mathbf{x})$ is a real-valued function and is greater than or equal to 0 aiming at measuring the constraint violation, $H$ is a function of the constraint violation, and $\mu$ defines a positive penalty parameter aiming at 
balancing objective and constraint violation. An individual point is feasible if $\zeta(\mathbf{x})=0$.

In constrained optimization, it is very important to find the right balance between the objective function and the constraint violation. The penalty function method can be applied to any type of constraints, but the performance of penalty-type methods is not always satisfactory. Usually, $\mu$ is updated throughout the iterative process, so that the sequence of the solutions of the unconstrained subproblems converges to the solution of the constrained problem. Small values of $\mu$ can produce almost optimal but infeasible solutions. On the other hand, large values of $\mu$ can give feasible solutions although an optimal solution in the boundary may not be found. Hence, the most difficult aspect of a penalty function method is to determine the appropriate value to initialize the parameter $\mu$, as well as the rule for its updating. For this reason alternative constraints handling techniques have been proposed in the last decades. Here, three different constraints handling techniques, usually used in population-based methods - stochastic ranking, global competitive ranking, and the feasibility and dominance rules - have been implemented, and extensively tested, in our m-CDE algorithm. They are briefly described below.

\subsection{Stochastic Ranking}

Runarsson and Yao (2000) first proposed stochastic ranking for the constrained optimization. This is a bubble-sort-like algorithm to give ranks to individuals in a population stochastically. In this ranking method, two adjacent individual points are compared and given ranks and swapped. The algorithm is halt if there is no swap. Individuals are ranked primarily based on their constraint violations. The objective function values are then considered if: i) individuals are feasible, or ii) a uniform random number between 0 and 1 is less than or equal to $P_{f}$. The probability $P_{f}$ is used only for comparisons of the objective function in the infeasible region of the search space.

That is, given any pair of two adjacent individual points, the probability of comparing them (in order to determine which one is fitter) according to the objective function is 1 if both individuals are feasible; otherwise it is $P_{f}$. Such ranking ensures that good feasible solutions as well as promising infeasible ones are ranked in the top of the population.

In our implementation of the stochastic ranking (SR) method (Runarsson and Yao, 2000) in the modified constrained differential evolution, each individual point $\mathbf{x}_{i}$ is evaluated according to the fitness function

$$
\Phi_{\mathrm{SR}}\left(\mathbf{x}_{i}\right)=\frac{I_{i}-1}{N-1},
$$

where $I_{i}$ represents the rank of the point $\mathbf{x}_{i}$ of the population and $N$ is the number of individuals in a population. From (3), the fitness of an individual point having the highest rank will be 0 and that with the lowest rank will be 1 . The best individual point in a population has the lowest fitness value.

\subsection{Global Competitive Ranking}

Runarsson and Yao (2003) proposed another constraints handling technique for constrained problems in order to strike the right balance between the objective function and the constraint violation. This method is called global competitive ranking. In this method, an individual point is ranked by comparing it against all other members of the population.

In this ranking process, after calculating $f$ and $\zeta$ for all the individuals, $f$ and $\zeta$ are sorted separately in ascending order (since we consider the minimization problem) and given ranks. Special consideration is given to the tied individuals. In the case of tied individuals the same higher rank will be given. For example, in these eight individuals, already in ascending order, $\langle 6,(5,8), 1,(2,4,7), 3\rangle$ (individuals in parentheses have same value) the corresponding ranks are $I(6)=1, I(5)=I(8)=2, I(1)=4, I(2)=I(4)=$ $I(7)=5, I(3)=8$. After the ranking of all the individuals based on the objective function $f$ and the constraint violation $\zeta$, separately, the fitness function of each individual point $\mathbf{x}_{i}$ is given by

$$
\Phi_{\mathrm{GR}}\left(\mathbf{x}_{i}\right)=P_{f} \frac{I_{i, f}-1}{N-1}+\left(1-P_{f}\right) \frac{I_{i, \zeta}-1}{N-1},
$$

where $\Phi_{\mathrm{GR}}$ means fitness based on the global competitive ranking (GR), and $I_{i, f}$ and $I_{i, \zeta}$ are the ranks of point $\mathbf{x}_{i}$ based on the objective function and the constraint violation, respectively. $P_{f}$ indicates the probability that the fitness is calculated based on the rank of objective function. It is clear from the above that $P_{f}$ can be used easily to bias the calculation of fitness according to the objective function or the average constraint violation. The probability should take a value $0.0<P_{f}<0.5$ in order to guarantee that a feasible solution may be found. From (4), the fitness of an individual point is a value between 0 and 1 , and the best individual point in a population has the lowest fitness value.

\subsection{Feasibility and Dominance Rules}

Deb (2000) proposed another constraints handling technique for population-based solution methods. It 
is based on a set of rules that use feasibility and dominance (FD) principles, as follows. First, the constraint violation $\zeta$ is calculated for all the individuals in a population. Then the objective function $f$ is evaluated only for feasible individuals. Two individuals are compared at a time, and the following criteria are always enforced:

1. any feasible point is preferred to any infeasible point;

2. between two feasible points, one having better objective function is preferred;

3. between two infeasible points, one having smaller constraint violation is preferred.

In this case, the fitness of each individual point $\mathbf{x}_{i}$ is calculated as follows

$$
\Phi_{\mathrm{FD}}\left(\mathbf{x}_{i}\right)= \begin{cases}f\left(\mathbf{x}_{i}\right) & \text { if } \mathbf{x}_{i} \text { is feasible } \\ f_{\max , f}+\zeta\left(\mathbf{x}_{i}\right) & \text { otherwise, }\end{cases}
$$

where $f_{\max , f}$ is the objective function of the worst feasible solution in the population. When all individuals are infeasible then its value is set to zero. This fitness function is used to choose the best individual point in a population.

\section{Modified Constrained Differential Evolution}

The population-based differential evolution algorithm has become popular and has been used in many practical cases, mainly because it has demonstrated good convergence properties and is easy to understand. DE is a floating point encoding that creates a new candidate point by adding the weighted difference between two individuals to a third one in the population. This operation is called mutation. The mutant point's components are then mixed with the components of target point to yield the trial point. This mixing of components is referred to as crossover. In selection, a trial point replaces a target point for the next generation only if it is considered an equal or better point. In unconstrained optimization, the selection operation relies on the objective function. DE has three parameters: amplification factor of differential variation $F$, crossover control parameter $\mathrm{Cr}$, and population size $N$.

It is not an easy task to set the appropriate parameters since these depend on the nature and size of the optimization problems. Hence, self-adaptive control parameters ought to be implemented. Brest et al. (2006) proposed self-adaptive control parameters for DE when solving global optimization problems with simple bounds. In most original DE, three points are chosen randomly for mutation and the base point is then chosen at random within the three. This has an exploratory effect but it slows down the convergence of DE. Kaelo and Ali (2006) proposed a modified mutation for differential evolution.

The herein presented modified constrained differential evolution algorithm - m-CDE - for constrained nonlinear programming problems (1) includes:

1. the self-adaptive control parameters $F$ and $\mathrm{Cr}$, as proposed by Brest et al. (2006);

2. a modified mutation that mixes the modification proposed by Kaelo and Ali (2006) with the cyclical use of the overall best point as base point;

3 . the inversion operation;

4. a modified selection that is based on the fitness of individuals;

5. the elitism.

The modification in mutation allows m-CDE to enhance the local search around the overall best point. In modified selection of $\mathrm{m}-\mathrm{CDE}$, we introduce and test the three different techniques described so far for calculating the fitness function of individuals that are capable to handle the constrained problems (1). The modified constrained differential evolution is outlined below.

The target point of $\mathrm{m}-\mathrm{CDE}$, at iteration/generation $z$, is defined by $\mathbf{x}_{i, z}=\left(x_{i 1, z}, x_{i 2, z}, \ldots, x_{i n, z}\right)$, where $n$ is the number of variables of the optimization problem and $i=1,2, \ldots, N . N$ does not change during the optimization process. The initial population is chosen randomly and should cover the entire component spaces.

Self-adaptive control parameters: In m-CDE, we use self-adaptive control parameters for $\mathrm{F}$ and $\mathrm{Cr}$ proposed by Brest et al. (2006) by generating a different set $\left(F_{i}, C r_{i}\right)$ for each point $\mathbf{x}_{i}$ in the population. The new control parameters for next generation $F_{i, z+1}$ and $C r_{i, z+1}$ are calculated by

$$
\begin{aligned}
& F_{i, z+1}= \begin{cases}F_{l}+\lambda_{1} \times F_{u} & \text { if } \lambda_{2}<\tau_{1} \\
F_{i, z} & \text { otherwise }\end{cases} \\
& C r_{i, z+1}= \begin{cases}\lambda_{3} & \text { if } \lambda_{4}<\tau_{2} \\
C r_{i, z} & \text { otherwise, }\end{cases}
\end{aligned}
$$

where $\lambda_{k} \sim \mathrm{U}[0,1], k=1, \ldots, 4$ and $\tau_{1}=\tau_{2}=0.1$ represent the probabilities to adjust parameters $F_{i}$ and $C r_{i}$, respectively. $F_{l}=0.1$ and $F_{u}=0.9$, so the new $F_{i, z+1}$ takes a value from $[0.1,1.0]$ in a random manner. The new $C r_{i, z+1}$ takes a value from $[0,1] . F_{i, z+1}$ and $C r_{i, z+1}$ are obtained before the mutation is performed. So, they influence the mutation, crossover and selection operations of the new point $\mathbf{x}_{i, z+1}$.

Modified mutation: In $\mathrm{m}-\mathrm{CDE}$, this is a mixture of two different types of mutation operations. We use 
the mutation proposed in (Kaelo and Ali, 2006). After choosing three points randomly, the best point among three based on the fitness function is selected for the base point and the remaining two points are used as differential variation, i.e., for each target point $\mathbf{x}_{i, z}$, a mutant point is created according to

$$
\mathbf{v}_{i, z+1}=\mathbf{x}_{r_{3}, z}+F_{i, z+1}\left(\mathbf{x}_{r_{1}, z}-\mathbf{x}_{r_{2}, z}\right),
$$

where $r_{1}, r_{2}, r_{3}$ are randomly chosen from the set $\{1,2, \ldots, N\}$, mutually different and different from the running index $i$ and $r_{3}$ is the index with the best fitness (among the three points). This modification has a local effect when the points of the population form a cluster around the global minimizer.

Furthermore, at every $B$ generations, the best point found so far is used as the base point and two randomly chosen points are used as differential variation, i.e.,

$$
\mathbf{v}_{i, z+1}=\mathbf{x}_{\text {best }}+F_{i, z+1}\left(\mathbf{x}_{r_{1}, z}-\mathbf{x}_{r_{2}, z}\right) .
$$

These mixed modifications allow $\mathrm{m}-\mathrm{CDE}$ to maintain its exploratory feature and at the same time to exploit the region around the best individual of the population expediting the convergence.

Crossover: In order to increase the diversity of the mutant points' components, crossover is introduced. To this end, the crossover point $\mathbf{u}_{i, z+1}$ is formed, where

$$
u_{i j, z+1}= \begin{cases}v_{i j, z+1} & \text { if }\left(r_{j} \leq C r_{i, z+1}\right) \text { or } j=s_{i} \\ x_{i j, z} & \text { if }\left(r_{j}>C r_{i, z+1}\right) \text { and } j \neq s_{i}\end{cases}
$$

In (9), $r_{j} \sim \mathrm{U}[0,1]$ performs the mixing of $j$ th component of points, $s_{i}$ is randomly chosen from the set $\{1,2, \ldots, n\}$ and ensures that $\mathbf{u}_{i, z+1}$ gets at least one component from $\mathbf{v}_{i, z+1}$.

Inversion: Since in $\mathrm{m}-\mathrm{CDE}$, a point has $n$ dimensional real components, inversion (Holland, 1975) can easily be applicable. With the inversion probability $\left(p_{\text {inv }} \in[0,1]\right)$, two positions are chosen on the point $\mathbf{u}_{i}$, the point is cut at those positions, and the cut segment is reversed and reinserted back into the point to create the trial point $\mathbf{u}_{i}^{\prime}$. In practice, m$\mathrm{CDE}$ with the inversion has been shown to give better results than those obtained without the inversion. An illustrative example of inversion is shown in Figure 1.

Bounds check: When generating the mutant point and when the inversion operation is performed, some components can be generated outside the bound constraints. So, in m-CDE after inversion the bounds of each component should be checked with the following projection of bounds:

$$
u_{i j, z+1}^{\prime}= \begin{cases}l_{j} & \text { if } u_{i j, z+1}^{\prime}<l_{j} \\ u_{j} & \text { if } u_{i j, z+1}^{\prime}>u_{j} \\ u_{i j, z+1}^{\prime} & \text { otherwise }\end{cases}
$$

Modified selection: In original DE, the target and the trial points are compared based on their corresponding objective function to decide which point becomes a member of the next generation, that is if the trial point's objective function is less than or equal to the that of target point, then the trial point will be the target point for the next generation.

In this paper, for constrained nonlinear programming problems, we propose a modified selection based on one of the fitness functions of individuals discussed so far. When using the global competitive ranking technique, all the target points at generation $z$ and trial points at generation $z+1$ are ranked together and their corresponding fitness $\Phi_{\mathrm{GR}}$ are calculated. Then the modified selection is performed, i.e., the trial and the target points are compared to decide which will be the new target points for next generation based on their calculated fitness by the following way

$$
\mathbf{x}_{i, z+1}= \begin{cases}\mathbf{u}_{i, z+1}^{\prime} & \text { if } \Phi_{\mathrm{GR}}\left(\mathbf{u}_{i, z+1}^{\prime}\right) \leq \Phi_{\mathrm{GR}}\left(\mathbf{x}_{i, z}\right) \\ \mathbf{x}_{i, z} & \text { otherwise }\end{cases}
$$

After performing selection in $\mathrm{m}-\mathrm{CDE}$, the best point is chosen in the current generation based on the lowest fitness function of the target points.

A similar procedure is performed when the stochastic ranking technique is implemented.

On the other hand, when using the feasibility and dominance principles, the trial and the target points are compared based on the three feasibility and dominance rules to decide which will be the new target points for next generation. After performing selection, the fitness function $\Phi_{\mathrm{FD}}$ for all the target points are calculated, and the best point based on the lowest fitness function in the current generation is chosen. We remark that this point is the overall best point in the entire generations so far.

Elitism: The elitism is also performed to keep the best point found so far in the entire generations. The elitism aims at preserving in the entire generations the individual point that, with the constraint violation 0 or smaller than others, has the smaller objective function. This is only required when either the stochastic ranking or the global competitive ranking is used to calculate fitness of individuals. We remark that in these two techniques, fitness values of individuals are calculated at every generation based on their corresponding ranks. Thus, the fitness of best individual point (based on the objective function and the constraint violation) may not be the lowest one.

Termination criterion: Let $G_{\max }$ be the maximum number of generations. If $f_{\max , z}$ and $f_{\min , z}$ are the objective function values of the points that have the 


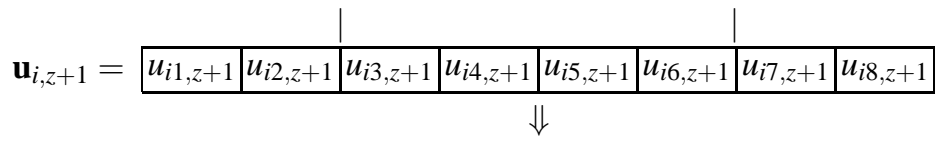

$$
\begin{aligned}
& \begin{array}{ll|l|l|l|l|l|l|l|}
\mathbf{u}_{i, z+1}^{\prime} & \multicolumn{4}{|l|}{} \\
\hline u_{i 1, z+1} & u_{i 2, z+1} & u_{i 6, z+1} & u_{i 5, z+1} & u_{i 4, z+1} & u_{i 3, z+1} & u_{i 7, z+1} & u_{i 8, z+1} \\
\hline
\end{array}
\end{aligned}
$$

Figure 1: Inversion used in $\mathrm{m}-\mathrm{CDE}$

highest and the lowest fitness function values respectively, attained at generation $z$, then our $\mathrm{m}-\mathrm{CDE}$ algorithm terminates if $\left(z>G_{\max }\right.$ or $\left.\left(f_{\max , z}-f_{\min , z}\right) \leq \eta\right)$, for a small positive number $\eta$.

\subsection{The m-CDE Algorithm}

The algorithm of the herein proposed modified constrained differential evolution for constrained global optimization is described in the following:

Step 1 : Set the values of parameters $N, G_{\max }, B, P_{f}$, $F_{l}, F_{u}, \tau_{1}, \tau_{2}, p_{\text {inv }}$, and $\eta$.

Step 2 : Set $z=1$. Initialize the population $\mathbf{x}_{1}, F_{1}$ and $C r_{1}$.

Step 3 : Calculate the fitness function $\Phi\left(\mathbf{x}_{z}\right)$.

Step 4 : Choose $f_{\max , z}$ and $f_{\min , z}$ from target points. For stochastic and global competitive ranking, perform the elitism to choose $f_{\text {best }}$ and $\mathbf{x}_{\text {best }}$. Otherwise for feasibility and dominance rules technique, set $f_{\text {best }}=f_{\min , z}$ and $\mathbf{x}_{\text {best }}=\mathbf{x}_{\min , z}$.

Step 5 : If the termination criterion is met stop. Otherwise set $z=z+1$.

Step 6 : Compute the control parameters $F_{z}$ and $\mathrm{Cr}_{z}$.

Step 7 : Compute the mutant point $\mathbf{v}_{z}$ : If $\operatorname{MOD}(z, B)=0$; use (8). Otherwise use (7).

Step 8 : Perform the crossover to make point $\mathbf{u}_{z}$.

Step 9 : If a random number $\gamma \sim \mathrm{U}[0,1] \leq p_{\text {inv }}$, perform inversion to make trial point $\mathbf{u}_{z}^{\prime}$.

Step 10 : Check the bounds of the trial points.

Step 11 : Calculate the fitness function $\Phi\left(\mathbf{x}_{z}\right)$ for all the target and trial points.

Step 12 : Perform the modified selection discussed above.

Step 13 : Go to step 4.

\section{Experimental Results}

We code $\mathrm{m}-\mathrm{CDE}$ in $\mathrm{C}$ with AMPL (Fourer, Gay and Kernighan, 1993) interfacing and compile with Microsoft Visual Studio 9.0 compiler in a PC having 2.5 $\mathrm{GHz}$ Intel Core 2 Duo processor and 4 GB RAM.
We set the value of parameters $N=\min (100,10 n)$, $B=10, P_{f}=0.45, p_{\text {inv }}=0.05$ and $\eta=10^{-6}$. We consider 13 benchmark constrained nonlinear programming problems (Runarsson and Yao, 2000). The characteristics of these test problems are outlined in Table 1. We model these problems in AMPL modeling systems. To access these models please visit http://www.norg.uminho.pt/emgpf/problems.htm.

Table 1: Characteristics of the test problems.

\begin{tabular}{c|c|r|c|c|c|c}
\hline \multirow{2}{*}{ Prob. } & Type of & \multirow{2}{*}{$f_{\text {opt }}$} & \multicolumn{2}{|c|}{ Var. } & \multicolumn{3}{|c}{ Constraints } \\
\cline { 5 - 7 } & $f$ & & $n$ & $m_{1}$ & $m_{2}$ & $m$ \\
\hline g01 & quadratic & -15.0000 & 13 & 9 & 0 & 9 \\
g02 & general & -0.8036 & 20 & 2 & 0 & 2 \\
g03 & polynomial & -1.0005 & 10 & 0 & 1 & 1 \\
g04 & quadratic & -30665.5387 & 5 & 6 & 0 & 6 \\
g05 & cubic & 5126.4967 & 4 & 2 & 3 & 5 \\
g06 & cubic & -6961.8139 & 2 & 2 & 0 & 2 \\
g07 & quadratic & 24.3062 & 10 & 8 & 0 & 8 \\
g08 & general & -0.0958 & 2 & 2 & 0 & 2 \\
g09 & general & 680.6301 & 7 & 4 & 0 & 4 \\
g10 & linear & 7049.2480 & 8 & 6 & 0 & 6 \\
g11 & quadratic & 0.7499 & 2 & 0 & 1 & 1 \\
g12 & quadratic & -1.0000 & 3 & 1 & 0 & 1 \\
g13 & general & 0.0539 & 5 & 0 & 3 & 3 \\
\hline
\end{tabular}

The following average measure of constraint violation of an individual point $\mathbf{x}$ is used:

$$
\zeta(\mathbf{x})=\frac{1}{m}\left(\sum_{k=1}^{m_{1}} \max \left\{0, g_{k}(\mathbf{x})\right\}+\sum_{l=1}^{m_{2}}\left|h_{l}(\mathbf{x})\right|\right),
$$

where $m=m_{1}+m_{2}$ is the total number of constraints. In this paper, we consider an individual point as a feasible one if $\zeta(\mathbf{x}) \leq \delta$, where $\delta$ is a very small positive number. Here we set $\delta=10^{-8}$.

We test $\mathrm{m}$-CDE with the previously described constraints handling techniques and compare their performance using performance profiles (Dolan and Moré, 2002). A comparison with other solution methods found in literature is also included.

\subsection{Performance Profiles}

For a fair comparison we run all the variants of m$\mathrm{CDE}$ in comparison for 30 times and reported the results. We used different $G_{\max }$ for the 13 problems, but used the same value for all the variants. 
We used the performance profiles proposed by Dolan and Moré (2002) to evaluate and compare the performance of the variants. Performance profiles are the graphical representation of performance of different solvers/variants/codes for a set of test problems on the basis of their performance ratio. The performance profile plot represents the cumulative distribution function of the performance ratio based on an appropriate performance metric. The authors proposed the computing time required to solve a problem as a performance metric for different solvers (Dolan and Moré, 2002), but other performance metrics could be used.

In our comparative study, let $\mathcal{P}$ be the set of all problems and $\mathcal{S}$ be the set of all variants of m-CDE. Also let $m_{(p, s)}$ be the performance metric found by variant $s \in \mathcal{S}$ on problem $p \in \mathcal{P}$ that measures the relative improvement of the objective function values, a scaled distance to the optimal objective function value $f_{\text {opt }}$ (Ali, Khompatraporn and Zabinsky, 2005), defined by

$$
m_{(p, s)}=\frac{f_{(p, s)}-f_{\mathrm{opt}}}{f_{w}-f_{\mathrm{opt}}} .
$$

In (12), $f_{(p, s)}$ is the average/best of objective function values found by variant $s$ on problem $p$ after 30 runs and $f_{w}$ is the worst objective function value of problem $p$ after 30 runs among all variants. Since a zero value of $\min \left\{m_{(p, s)}: s \in \mathcal{S}\right\}$ may appear, the performance ratios used in our comparative study are defined (Vaz and Vicente, 2007) by

$$
r_{(p, s)}= \begin{cases}1+m_{(p, s)}-q & \text { if } q \leq 10^{-5} \\ \frac{m_{(p, s)}}{q} & \text { otherwise, }\end{cases}
$$

where $q=\min \left\{m_{(p, s)}: s \in \mathcal{S}\right\}$. Then $\rho_{s}(\tau)$, the fraction of problems for which the variant $s$ has a performance ratio $r_{(p, s)}$ within a factor $\tau$, is given by

$$
\rho_{s}(\tau)=\frac{n_{P_{\tau}}}{n_{P}}
$$

where $n_{P_{\tau}}$ is the number of problems in $\mathcal{P}$ with $r_{(p, s)} \leq$ $\tau$ and $n_{P}$ is the total number of problems in $\mathcal{P}$. $\rho_{s}(\tau)$ is the probability (for variant $s \in \mathcal{S}$ ) that the performance ratio $r_{(p, s)}$ is within a factor $\tau \in \mathbb{R}$ of the best possible ratio.

At first, we tested our program with the stochastic ranking and the global competitive rankings for obtaining solutions of the above 13 constrained problems to check which technique is better than the others in comparison. The variants were stochastic ranking, global competitive ranking 1 , with a fixed value of $P_{f}$, and global competitive ranking 2, with a random value of $P_{f}$ in the interval $(0,0.45)$. Figure 2 shows the profiles of the performance metric $f_{\text {avg }}$, the average of the best solutions obtained over the 30 runs. If we are only interested in knowing which vari-

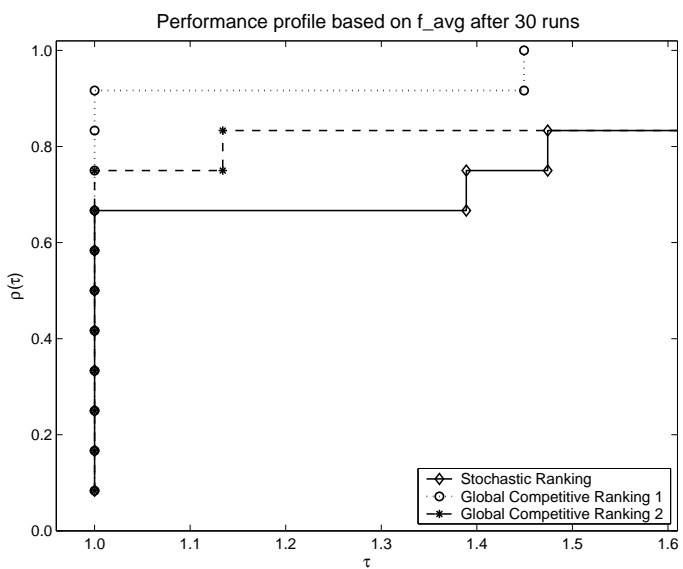

Figure 2: Performance profile of average objective function value for different variants.

ant is the most efficient, in the sense that it reaches the best solutions mostly, we compare the values of $\rho$ for $\tau=1$, and find the highest value which is the probability that the variant will win over the remaining ones. However, to know the robustness of variants, we compare the values of $\rho(\tau)$ for large values of $\tau$. It means that variants with the largest probabilities $\rho(\tau)$ for large values of $\tau$ are the most robust ones. In this figure it is shown that variant global competitive ranking 1 (for fixed value of $P_{f}$ ) wins over the other two variants of $\mathrm{m}-\mathrm{CDE}$.

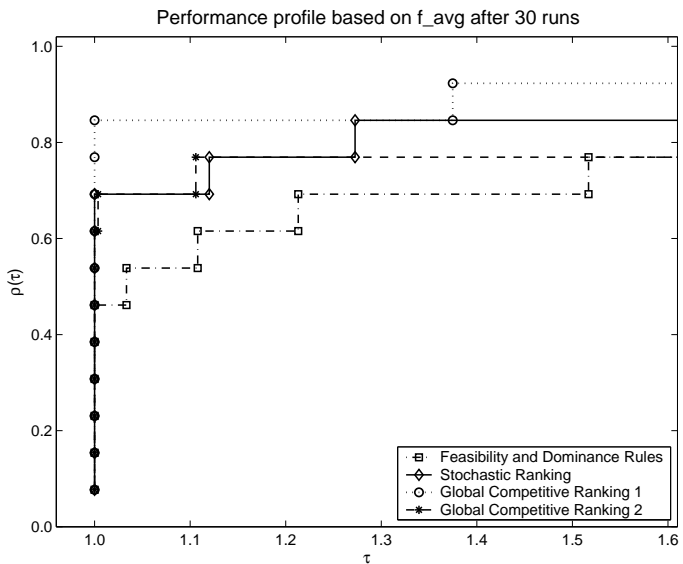

Figure 3: Performance profile of average objective function value using fitness (5).

In the second experiment, we tested the following four variants of m-CDE: feasibility and dominance rules, stochastic ranking, global competitive ranking 1 and global competitive ranking 2 (as previously defined). To be able to fairly compare with the variant feasibility and dominance rules, the other variants were fairly modified. After the modified se- 
Table 2: Experimental results of the 13 test problems by m-CDE1.

\begin{tabular}{c|r|r|r|r|r|r|r}
\hline Prob. & \multicolumn{1}{|c|}{ best } & \multicolumn{1}{c|}{ worst } & \multicolumn{1}{c}{ avg. } & \multicolumn{1}{c}{ median } & std. dev. & avg. vio. & $G_{\text {avg }}$ \\
\hline g01 & -15.0000 & -15.0000 & -15.0000 & -15.0000 & $1.16 \mathrm{E}-06$ & $0.00 \mathrm{E}+00$ & 196 \\
g02 & -0.8036 & -0.7926 & -0.8007 & -0.8036 & $4.95 \mathrm{E}-03$ & $0.00 \mathrm{E}+00$ & 1408 \\
g03 & -1.0000 & -0.9998 & -1.0000 & -1.0000 & $3.90 \mathrm{E}-05$ & $5.88 \mathrm{E}-09$ & 1750 \\
g04 & -30665.5387 & -30665.5387 & -30665.5387 & -30665.5387 & $2.38 \mathrm{E}-05$ & $8.66 \mathrm{E}-09$ & 1091 \\
g05 & 5126.4978 & 5126.4986 & 5126.4979 & 5126.4978 & $1.83 \mathrm{E}-04$ & $9.23 \mathrm{E}-09$ & 1750 \\
g06 & -6961.8161 & -6624.1610 & -6950.5609 & -6961.8161 & $6.16 \mathrm{E}+01$ & $9.67 \mathrm{E}-09$ & 1750 \\
g07 & 24.2316 & 24.2319 & 24.2317 & 24.2316 & $7.44 \mathrm{E}-05$ & $1.60 \mathrm{E}-09$ & 1750 \\
g08 & -0.0958 & -0.0958 & -0.0958 & -0.0958 & $2.71 \mathrm{E}-06$ & $0.00 \mathrm{E}+00$ & 42 \\
g09 & 680.6301 & 680.6301 & 680.6301 & 680.6301 & $1.38 \mathrm{E}-06$ & $0.00 \mathrm{E}+00$ & 727 \\
g10 & 7049.2533 & 7076.5860 & 7053.3441 & 7050.6330 & $6.99 \mathrm{E}+00$ & $1.13 \mathrm{E}-09$ & 1750 \\
g11 & 0.7500 & 0.7670 & 0.7506 & 0.7500 & $3.11 \mathrm{E}-03$ & $6.36 \mathrm{E}-09$ & 158 \\
g12 & -1.0000 & -1.0000 & -1.0000 & -1.0000 & $2.33 \mathrm{E}-06$ & $0.00 \mathrm{E}+00$ & 30 \\
g13 & 0.0539 & 0.0539 & 0.0539 & 0.0539 & $3.53 \mathrm{E}-17$ & $9.96 \mathrm{E}-09$ & 1750 \\
\hline
\end{tabular}

lection step of the algorithm, the fitness function is recalculated now using (5) so that the best and the worst target points of the population are identified according to the objective function and constraint violation values. Figure 3 shows the profiles of the performance metric $f_{\text {avg }}$ for the four variants. In this figure it is shown that variant global competitive ranking 1 here also wins over the other three variants of $\mathrm{m}-\mathrm{CDE}$ in comparison.

From the above discussion it is clear that in both cases the variant of $\mathrm{m}-\mathrm{CDE}$ based on global competitive ranking with fixed value of $P_{f}$ gave better performance over the other ones. We also tested our m-CDE with global dense ranking technique but the obtained results were significantly worse than those obtained by global competitive ranking technique.

\subsection{Comparing With Other Methods}

We also compare our m-CDE (based on the global competitive ranking with fixed value of $P_{f}$ ) with the stochastic ranking presented in (Runarsson and Yao, 2000) and the global competitive ranking presented in (Runarsson and Yao, 2003). The authors proposed these techniques based on a $(30,200)$ evolution strategy. Here, the stochastic ranking technique is denoted by SRES and the global competitive ranking technique is GRES. An adaptive penalty scheme for constraint handling with dynamic use of variants of differential evolution (DUVDE) (Silva et al., 2011) is also used in the comparison. We set the same value of maximum number of generations according to (Runarsson and Yao, 2000, 2003). We set $G_{\max }=1750$ for all the problems except problem g12 for all the runs. $G_{\max }=175$ was set for $\mathrm{g} 12$. Here, we aim to get a solution within $0.001 \%$ of the optimal solution $f_{\text {opt }}$.

Firstly, we run all the 13 problems 30 times with the global competitive ranking technique using modi- fied constrained differential evolution. Here we identified $f_{\min }$ and $f_{\max }$ of the objective function based on the current fitness function $\Phi_{\mathrm{GR}}$ at generation $z$. Then elitism was performed to determine the $f_{\text {best }}$. Hereafter we will denote this version by m-CDE1. We report 'best', 'worst', 'avg.' (average), 'median' and 'std. dev.' (standard deviation) of the best objective functions and 'avg. vio.' (average constraint violation) among all the 30 runs. The average number of generations ' $G_{\text {avg }}$ ' attained among the 30 runs is also reported. These results are shown in Table 2.

Secondly, we run again all the 13 problems 30 times with the global competitive ranking technique using modified constrained differential evolution, but this time, both $f_{\min }$ and $f_{\max }$ are identified after recalculating the fitness of all individuals in the population using function $\Phi_{\mathrm{FD}}$, at each generation $z$. Then we set $f_{\text {best }}=f_{\text {min }}$. Hereafter we will denote this version by $\mathrm{m}-\mathrm{CDE} 2$. We remark that $\mathrm{m}-\mathrm{CDE} 2$ is the variant global competitive ranking 1 of the second experience reported in the previous subsection. The results are shown in Table 3. It is shown from Tables 2 and 3 that in almost all problems with respect to the different objective function measures the results obtained by $\mathrm{m}-\mathrm{CDE} 1$ are relatively better than those of m-CDE2.

Finally, to compare the results obtained by mCDE1, shown in Table 2, with DUVDE, SRES and GRES, results of 'best', 'avg.' and 'std. dev.' of the best objective functions among 30 runs are shown in Table 4. These results are taken from the cited papers (Silva et al., 2011), (Runarsson and Yao, 2000) and (Runarsson and Yao, 2003) respectively. In m-CDE1, we use the population size $N$ dependent on the dimension of test problem and use the maximum number of generations according to (Runarsson and Yao, 2000, 2003), although in DUVDE the authors used the population size 50 and the maximum number of generations 3684 for all the tested problems. Problems g12 
Table 3: Experimental results of the 13 test problems by m-CDE2.

\begin{tabular}{c|r|r|r|r|r|r|r}
\hline Prob. & \multicolumn{1}{|c|}{ best } & \multicolumn{1}{c|}{ worst } & \multicolumn{1}{c|}{ avg. } & \multicolumn{1}{c}{ median } & std. dev. & avg. vio. & $G_{\text {avg }}$ \\
\hline g01 & -15.0000 & -15.0000 & -15.0000 & -15.0000 & $1.22 \mathrm{E}-06$ & $0.00 \mathrm{E}+00$ & 196 \\
g02 & -0.8036 & -0.7926 & -0.8004 & -0.8036 & $5.03 \mathrm{E}-03$ & $2.07 \mathrm{E}-10$ & 1438 \\
g03 & -1.0000 & -0.9966 & -0.9997 & -0.9999 & $6.53 \mathrm{E}-04$ & $4.54 \mathrm{E}-09$ & 1750 \\
g04 & -30665.5387 & -30665.5387 & -30665.5387 & -30665.5387 & $2.47 \mathrm{E}-05$ & $8.24 \mathrm{E}-09$ & 1039 \\
g05 & 5126.4978 & 5126.4978 & 5126.4978 & 5126.4978 & $1.85 \mathrm{E}-12$ & $1.00 \mathrm{E}-08$ & 1750 \\
g06 & -6961.8161 & -6615.7067 & -6950.2790 & -6961.8161 & $6.32 \mathrm{E}+01$ & $9.94 \mathrm{E}-09$ & 1695 \\
g07 & 24.2316 & 24.2325 & 24.2318 & 24.2317 & $1.98 \mathrm{E}-04$ & $1.75 \mathrm{E}-09$ & 1750 \\
g08 & -0.0958 & -0.0958 & -0.0958 & -0.0958 & $2.86 \mathrm{E}-06$ & $0.00 \mathrm{E}+00$ & 30 \\
g09 & 680.6301 & 680.6301 & 680.6301 & 680.6301 & $1.10 \mathrm{E}-06$ & $0.00 \mathrm{E}+00$ & 730 \\
g10 & 7049.2604 & 7250.9916 & 7100.8072 & 7051.2523 & $7.57 \mathrm{E}+01$ & $1.38 \mathrm{E}-09$ & 1750 \\
g11 & 0.7500 & 1.0000 & 0.9353 & 1.0000 & $1.09 \mathrm{E}-01$ & $1.74 \mathrm{E}-09$ & 1515 \\
g12 & -1.0000 & -1.0000 & -1.0000 & -1.0000 & $2.64 \mathrm{E}-06$ & $0.00 \mathrm{E}+00$ & 20 \\
g13 & 0.0539 & 0.0539 & 0.0539 & 0.0539 & $1.14 \mathrm{E}-05$ & $9.70 \mathrm{E}-09$ & 1750 \\
\hline
\end{tabular}

Table 4: Results from DUVDE, SRES and GRES.

\begin{tabular}{|c|c|c|c|c|c|c|c|c|c|}
\hline \multirow{2}{*}{ Prob. } & \multicolumn{3}{|c|}{ DUVDE } & \multicolumn{3}{|c|}{ SRES } & \multicolumn{3}{|c|}{ GRES } \\
\hline & best & avg. & std. dev. & best & avg & std. dev. & best & avg. & std. dev. \\
\hline g01 & -15.0000 & -12.5000 & $2.37 \mathrm{E}+00$ & -15.0000 & -15.0000 & $0.00 \mathrm{E}+00$ & -15.0000 & - & $0.00 \mathrm{E}+00$ \\
\hline g02 & -0.8036 & -0.7688 & $3.57 \mathrm{E}-02$ & -0.8035 & -0.7820 & $2.00 \mathrm{E}-02$ & -0.8035 & - & $1.70 \mathrm{E}-02$ \\
\hline $\mathrm{g} 03$ & -1.0000 & -0.2015 & $3.45 \mathrm{E}-01$ & -1.0000 & -1.0000 & $1.90 \mathrm{E}-04$ & -1.0000 & - & $2.60 \mathrm{E}-05$ \\
\hline g04 & -30665.5000 & -30665.5000 & $0.00 \mathrm{E}+00$ & -30665.5390 & -30665.5390 & $2.00 \mathrm{E}-05$ & -30665 & - & $5.40 \mathrm{E}-01$ \\
\hline g05 & 5126.4965 & 5126.4965 & $0.00 \mathrm{E}+00$ & 126.4970 & 5128.8810 & $3.50 \mathrm{E}+00$ & 5126. & - & $1.10 \mathrm{E}+00$ \\
\hline g06 & -6961.8000 & -6961.8000 & $0.00 \mathrm{E}+00$ & -6961.8140 & -6875.9400 & $1.60 \mathrm{E}+02$ & -6943.5600 & - & $2.90 \mathrm{E}+02$ \\
\hline g07 & 24.3060 & 30.4040 & $2.16 \mathrm{E}+01$ & 24.3070 & 24.3740 & $6.60 \mathrm{E}-02$ & 24.3 & - & $1.10 \mathrm{E}-01$ \\
\hline g08 & -0.0958 & -0.0958 & $0.00 \mathrm{E}+00$ & -0.0958 & -0.0958 & $2.60 \mathrm{E}-17$ & -0.0958 & - & $2.60 \mathrm{E}-17$ \\
\hline g09 & 80.6300 & 680.6300 & $3.00 \mathrm{E}-05$ & 680.6300 & 680.6560 & $3.40 \mathrm{E}-02$ & 680.6310 & - & $5.80 \mathrm{E}-02$ \\
\hline $\mathrm{g} 10$ & 049.2500 & 7351.1700 & $5.26 \mathrm{E}+02$ & 7054.3160 & 7559.1920 & $5.30 \mathrm{E}+02$ & $*$ & - & * \\
\hline g11 & 0.7500 & 0.9875 & 5.59E-02 & 0.7500 & 0.7500 & 8.00E-05 & 500 & - & $7.20 \mathrm{E}-05$ \\
\hline g12 & $\dagger$ & $\dagger$ & $\dagger$ & -1.0000 & -1.0000 & $0.00 \mathrm{E}+00$ & -1.0000 & - & $0.00 \mathrm{E}+00$ \\
\hline g13 & $\dagger$ & $\dagger$ & $\dagger$ & 0.0539 & 0.0675 & $3.10 \mathrm{E}-02$ & 0.0539 & - & $1.30 \mathrm{E}-04$ \\
\hline
\end{tabular}

$(\dagger)$ not considered; (-) not available; $(*)$ not solved

and g13 were not tested with DUVDE (Silva et al., 2011). From Tables 2 and 4 we may conclude that for most of the problems, and with respect to all measures of comparison, m-CDE1 performs rather well when compared with DUVDE, SRES and GRES.

From the above discussion it is clear that the proposed modified constrained differential evolution based on the global competitive ranking technique to handle constraints, is rather effective when converging to constrained global solutions.

\section{Conclusions}

In this paper, to make the DE methodology more efficient to handle the constraints in constrained global optimization problems, a modified constrained differential evolution algorithm has been proposed. The modifications focus on self-adaptive control parameters, modified mutation, modified selection and elitism. Inversion operation has also been implemented in the proposed $\mathrm{m}-\mathrm{CDE}$.
To conclude, we emphasize the modifications that mostly influence the efficiency of the algorithm. The mixed modified mutation, in the m-CDE algorithm, aims at exploring both the entire search space (when using the mutation proposed in (Kaelo and Ali, 2006)) and the neighbourhood of the best point found so far (when using the best point as the base point cyclically). The modified selection, to handle the constraints effectively, uses a fitness function based on the global competitive ranking technique. In this technique, fitness of all target and trial points are calculated all together after ranking them based on the objective function and the constraint violation separately, for competing in modified selection to decide which points win for next generation population. This technique seems to have stricken the right balance between the objective function and the constraint violation for obtaining a global solution while satisfying the constraints.

To test the effectiveness of the new m-CDE, 13 benchmark constrained nonlinear programming problems have been considered. These problems have also 
been solved with the stochastic ranking and the feasibility and dominance rules techniques and comparison has been made based on their performance profiles. It is shown that $\mathrm{m}-\mathrm{CDE}$ with the global competitive ranking, based on a fixed value of $P_{f}$, is relatively better than the other two techniques. A comparison has also been made with other results from the literature: the adaptive penalty-based differential evolution, the stochastic ranking based on an evolution strategy, and the global competitive ranking based on an evolution strategy. It is shown that $\mathrm{m}-\mathrm{CDE}$ is rather competitive when compared with the other solution methods. Future developments will focus on the extension of the m-CDE to problems with mixed integer variables.

\section{ACKNOWLEDGEMENTS}

This work is supported by FCT (Fundação para a Ciência e a Tecnologia) and Ciência 2007, Portugal. We thank two anonymous referees for their valuable comments to improve this paper.

\section{REFERENCES}

Ali, M.M., Khompatraporn, C. and Zabinsky, Z.B. (2005). A numerical evaluation of several stochastic algorithms on selected continuous global optimization test problems. Journal of Global Optimization, 31, 635-672.

Barbosa, H.J.C. and Lemonge, A.C.C. (2003). A new adaptive penalty scheme for genetic algorithms. Information Sciences, 156, 215-251.

Brest, J., Greiner, S., Bošković, B., Mernik, M. and Žumer, V. (2006). Self-adapting control parameters in differential evolution: a comparative study on numerical benchmark problems. IEEE Transactions on Evolutionary Computation, 10, 646-657.

Coello Coello, C.A. (2000). Constraint-handling using an evolutionary multiobjective optimization technique. Civil Engineering and Environmental Systems, 17, 319346.

Coello Coello, C.A. and Cortés, N.C. (2004). Hybridizing a genetic algorithm with an artificial immune system for global optimization. Engineering Optimization, 36, 607634.

Deb, K. (2000). An efficient constraint handling method for genetic algorithms. Computer Methods in Applied Mechanics and Engineering, 186, 311-338.

Dolan, E.D. and Moré, J.J. (2002). Benchmarking optimization software with performance profiles. Mathematical Programming, 91, 201-213.

Dong, Y., Tang, J., Xu, B. and Wang, D. (2005). An application of swarm optimization to nonlinear programming.
Computers \& Mathematics with Applications, 49, 16551668.

Fletcher, R. and Leyffer, S. (2002). Nonlinear programming without a penalty function. Mathematical Programming, 91, 239-269.

Fourer, R., Gay, D.M. and Kernighan, B.W. (1993). AMPL: A modelling language for matematical programming. Boyd \& Fraser Publishing Co.: Massachusets.

Hedar, A.R. and Fukushima, M. (2006). Derivative-free filter simulated annealing method for constrained continuous global optimization. Journal of Global Optimization, $35,521-549$.

Holland, J.H. (1975). Adaptation in Natural and Artificial Systems. University of Michigan Press: Ann Arbor.

Kaelo, P. and Ali, M.M. (2006). A numerical study of some modified differencial evolution algorithms. European Journal of Operational Research, 169, 1176-1184.

Ray, T. and Tai, K. (2001). An evolutionary algorithm with a multilevel pairing strategy for single and multiobjective optimization. Foundations of Computing and Decision Sciences, 26, 75-98.

Ray, T. and Liew, K.M. (2003). Society and civilization: An optimization algorithm based on the simulation of social behavior. IEEE Transactions on Evolutionary Computation, 7, 386-396.

Rocha, A.M.A.C. and Fernandes, E.M.G.P. (2008). Feasibility and dominance rules in the electromagnetism-like algorithm for constrained global optimization. In Gervasi et al. (Eds.), Computational Science and Its Applications: Lecture Notes in Computer Science (vol. 5073, pp. 768-783). Springer: Heidelberg.

Rocha, A.M.A.C. and Fernandes, E.M.G.P. (2009). Self adaptive penalties in the electromagnetism-like algorithm for constrained global optimization problems. In Proceedings of the 8th World Congress on Structural and Multidisciplinary Optimization (pp. 1-10).

Runarsson, T.P. and Yao, X. (2000). Stochastic ranking for constrained evolutionary optimization. IEEE Transactions on Evolutionary Computation, 4, 284-294.

Runarsson, T.P. and Yao, X. (2003). Constrained evolutionary optimization - the penalty function approach. In Sarker et al. (Eds.), Evolutionary Optimization: International Series in Operations Research and Management Science (vol. 48, pp. 87-113), Springer: New York.

Silva, E.K., Barbosa, H.J.C. and Lemonge, A.C.C. (2011). An adaptive constraint handling technique for differential evolution with dynamic use of variants in engineering optimization. Optimization and Engineering, 12, 31-54.

Storn, R. and Price, K. (1997). Differential evolution a simple and efficient heuristic for global optimization over continuous spaces. Journal of Global Optimization, 11, 341-359.

Vaz, A.I.F. and Vicente, L.N. (2007). A particle swarm pattern search method for bound constrained global optimization. Journal of Global Optimization, 39, 197-219.

Zahara, E. and Hu, C.-H. (2008). Solving constrained optimization problems with hybrid particle swarm optimization. Engineering Optimization, 40, 1031-1049. 